\title{
Clinicopathological profile of ovarian tumors in the age group 10-20 years
}

\section{Priya V.*}

\author{
Department of Obstetrics and Gynecology, Government Medical College, Kottayam, Kerala, India
}

Received: 22 January 2017

Revised: 27 January 2017

Accepted: 03 February 2017

\author{
*Correspondence: \\ Dr. Priya V., \\ E-mail: Priya.drv@gmail.com
}

Copyright: $\odot$ the author(s), publisher and licensee Medip Academy. This is an open-access article distributed under the terms of the Creative Commons Attribution Non-Commercial License, which permits unrestricted non-commercial use, distribution, and reproduction in any medium, provided the original work is properly cited.

\begin{abstract}
Background: Adolescence is the period of transition from childhood to adulthood. $1 \%$ neoplasm occurs in this age group below 17 years, of this, 3\% are ovarian neoplasms. Aim was to study retrospectively the clinicopathological profile and outcome of ovarian tumors in the age group 10-20 years in Government Medical College, Kottayam, Kerala, India from January 2008 for six years.

Methods: Fifty Patients who have undergone laparotomy in this institution during the period were taken for study. Data regarding age, mode of presentation, diagnostic methods, treatment and histopathology were recorded. Followup was also taken into consideration. Patients with polycystic ovary, corpus luteal cyst, follicular cyst and endometriotic cyst are excluded.

Results: Majority of the patients fall between the age group 15-20 years. Major clinical presentation was pain (40\%), followed by abdominal mass. Laparotomy was done for all cases except three where laparoscopy was done. Imprint cytology taken for 3 cases. Histopathology showed malignant tumors in 6 cases and benign tumors in 44 cases. Epithelial tumors outnumbered germ cell tumors.

Conclusions: This study shows the predominance of epithelial neoplasms in the age group 10-20 years. Proportion of malignancy is $12 \%$.
\end{abstract}

Keywords: Imprint cytology, Laparotomy, Ovarian tumor

\section{INTRODUCTION}

Ovarian tumours account for the top five gynaecological malignancies in Indian women. ${ }^{1}$ About $50-60 \%$ of all cancers in India are related to cervix, breast, Corpus uteri and Ovaries. According to National Cancer Registry Programme report 2012-2014 in Regional cancer centre Trivandrum, breast is the leading site of tumour $(28.3 \%$ in females followed by thyroid gland $(13.9 \%)$, Cervix $(7.4 \%)$, ovary $(5.3 \%)$ and mouth $(3.9 \%) .^{2}$ Ovarian tumours are notorious for poor prognosis owing to the late detection of disease.

Adolescence is the period of transition from childhood to adulthood. Ovarian tumours are rare in adolescent age group. Ovarian neoplasm accounts for approximately $1 \%$ of all malignant tumours in girls aged 15 years. $^{3}$ Mature cystic teratomas are the most common benign tumours encountered. Chance of malignant transformation is $0.1-2 \%$. Malignant ovarian tumours in children and adolescent are rare accounting for $0.9 \%$ of all malignancies. ${ }^{4}$ Dysgerminoma is the most common malignant germ cell tumour.

Most of the patients will be presenting with pain. Pain may be due to torsion, intraperitoneal rupture, or bleeding in to ovarian tissue. Symptoms like pain nausea and vomiting should alert physician to rule out torsion. Most of these patients need emergency surgery. Diagnosis is 
mainly by clinical features, ultrasound, tumour markers, Doppler and CT scan.

Management of ovarian tumours in young is a dilemma for gynaecologists as preservation of uterus with adnexa for procreation is an important aspect of therapy. Surgery plays an important role in the treatment of tumours. Here various ovarian tumours in the age group 10- 20 years encountered in our department is highlighted.

\section{METHODS}

All cases of ovarian tumors occurring in age group 10-20 years who have undergone surgery in Government Medical College, Kottayam, Kerala, India during the prescribed period is collected and analyzed. Data regarding patient's age, symptoms, diagnosis, treatment, histopathology and follow up where ever possible is recorded.

\section{Exclusion criteria}

Polycystic ovary, follicular cyst, corpus luteal cyst, endometrioma.

\section{RESULTS}

Majority of patients belong to age group 15-20 years. Malignant tumors are seen at age of 11-13 years and 18 to 20 years. Out of 50 cases, $40 \%$ presented with pain abdomen, $38 \%$ with mass abdomen $14 \%$ with irregular bleeding $\mathrm{p} / \mathrm{v}$. Torsion ovary noted in $65 \%$ of patients presented with pain abdomen.

Table 1: Clinical presentation.

\begin{tabular}{|lll|}
\hline Presentation & Number & $\%$ \\
\hline Pain abdomen & 20 & $40 \%$ \\
\hline Mass abdomen & 19 & $38 \%$ \\
\hline Irregular bleeding PV & 7 & $14 \%$ \\
\hline Incidental & 4 & $8 \%$ \\
\hline
\end{tabular}

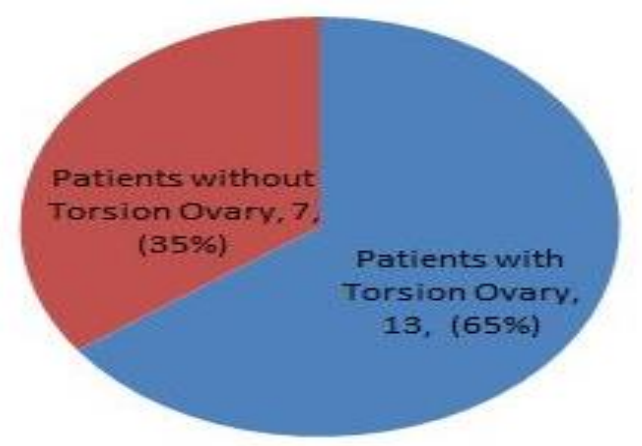

Figure 1: Percentage of torsion ovary in patients presented with pain abdomen.
Ultrasound revealed U/L cystic lesion in majority of cases. In two cases we had B/L involvement of the ovary. Solid ovarian lesion is encountered in fibrothecoma ovary and dysgerminoma. In malignant lesions, presence of ascites/ pleural effusion was also picked up by USG. Doppler showed low resistant flow pattern in malignant ovarian tumors and no vascularity especially in torsion ovaries. Tumour markers like CA-125 increased in fibrothecoma ovary and poorly differentiated carcinoma ovary. Carcino embryonic antigen is elevated in 2 cases of mucinous cystadeno carcinoma, Alphafeto protein is increased in yolk sac tumour. CA-19.9 elevated in immature teratoma grade II. FNAC from the mass showed epithelial neoplasm in two of malignant ovarian tumors and poorly differentiated carcinoma in one patient. CT is done in all patients with suspicious malignancy. Imprint cytology taken in 3 cases.

Majority of patients underwent laparotomy and laparoscopy was done for 3 patients. Ovarian cystectomy, Salpingo-ovariotomy and bilateral ovarian cystectomy are the surgical procedures done. Total abdominal hysterectomy with bilateral salpingo-oophorectomy with infracolic omentectomy done in 19-year-old patient with mucinous cystdeno-carcinoma.

Histopathology report shows $30 \%$ cases of serous cystadenoma, $24 \%$ cases of mucinous cystadenoma, $20 \%$ cases of teratoma, $2 \%$ cases of fibrothecoma and HPR non-conclusive in 6 cases due to torsion and gangrene where exact pathology could not be made out.

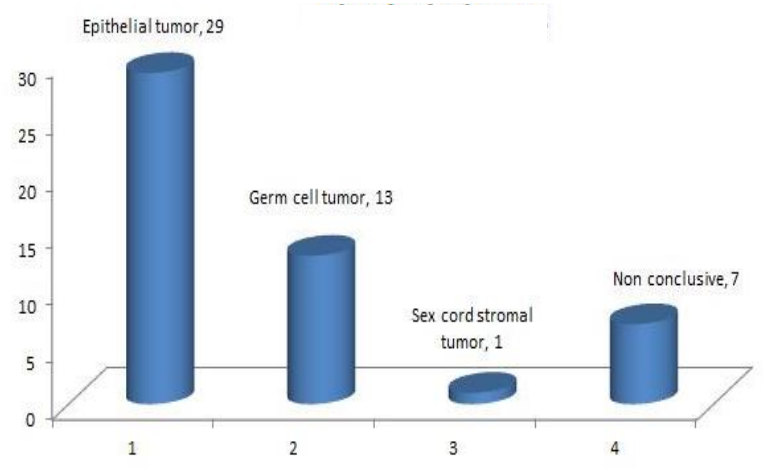

Figure 2: Distribution of ovarian neoplasm according to histological types.

In present study proportion of malignancy is $12 \%$. Among malignant tumors, we have 2 cases of mucinous cyst adenocarcinoma, 1 mixed germ cell tumour (dysgerminoma + endodermal sinus tumour), 1 immature teratoma grade 2, 1 yolk sac tumour, 1 poorly differentiated carcinoma.

Fallopian tube section is unremarkable in patient with yolk sac tumour. Fallopian tube is uninvolved by the neoplasm in patient who had TAH with BSO for mucinouscystadeno carcinoma. Patient with immature teratoma fallopian tube showed only congested vessels.. 


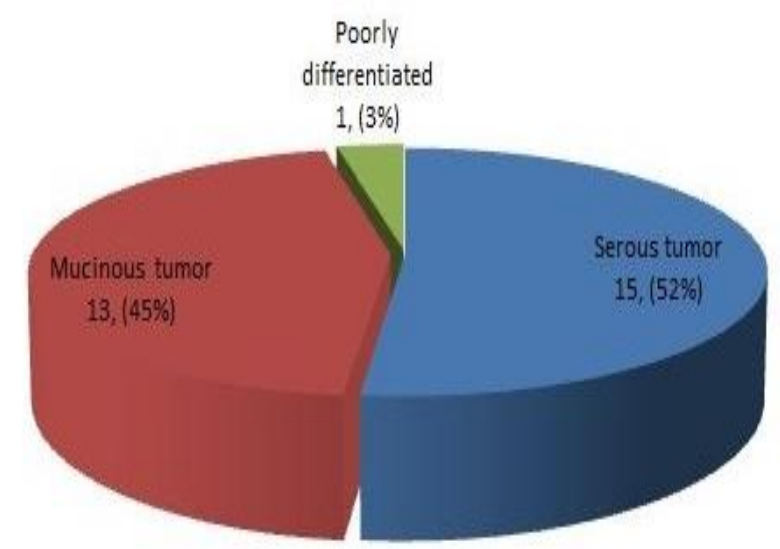

Figure3: Distribution of epithelial tumours.

Of the 6 patients one with poorly differentiated carcinoma, after one course of chemotherapy died. 11year-old patient with yolk sac tumour who had staging laparotomy and left salpingo-ovariotomy after 1st week of chemotherapy succumbed to death. One patient with mucinous cystadeno carcinoma who had TAH with BSO had 6 courses of chemotherapy and is now on follow up. She is alive now. Another patient with immature teratoma grade 2 had chemotherapy and now on follow up. Other 2 patients, one with mucinous cystadeno-carcinoma and one with mixed germ cell tumour, follow up details are not available.

\section{DISCUSSION}

Ovarian tumours are rare in adolescent age group. Most ovarian tumours in adolescents are benign, but possibility of malignancy is high. In a study of 25 years conducted by department of pathology (1990-2014), Delhi University by Rathore $\mathrm{R}$ showed pain abdomen as the most common presenting symptom in adolescent ovarian tumour followed by abdominal distension. ${ }^{5}$ We also had similar results. According to a study of incidence of ovarian tumours below 20 years in IMCH, Calicut showed $76 \%$ benign tumours, $35 \%$ germ cell tumours and $47 \%$ epithelial tumours. ${ }^{6}$ Present study revealed $26 \%$ germ cell tumours and $58 \%$ epithelial ovarian tumours and 1 benign sex cord stromal tumour (2\%). A ten-year study from the Department of pathology, North Bengal Medical College to find out the incidence of different ovarian tumours of girls up to 20 years, out of 151 cases $22.6 \%$ were malignant. Among malignant tumours $66 \%$ were germ cell tumours. There were 9 cases of malignant surface epithelial tumour. ${ }^{7}$

In the prospective study conducted at the Department of Obstetrics and Gynaecology, Tribhuvan University, 80 cases in childhood and adolescent were studied, majority belonged to 15-19 years. Germ cell tumours account for $73.7 \%$. Dermoid was the frequently occurring tumour. $23.7 \%$ of the total were malignant. ${ }^{8}$ Adolescent ovarian tumours, clinicopathological review from Pakistan,
Choudhary A et al showed that majority belonged to age group 14-16 years. Four cases were malignant. $70 \%$ of ovarian tumours were benign. Germ cell tumours were predominant and higher percentage of malignancies with in germ cell tumours in young children and adolescent. ${ }^{9}$ This is contradictory to present study in which epithelial ovarian tumours outnumber germ cell tumours and malignancy is seen both in germ cell and epithelial cell. Hernon MVK studied histology and management of ovarian cyst in children and adolescent from 1991-2007 in the Department of women's health, U.K. Benign teratoma was the common tumour encountered in the study. Malignant tumours include Granulosa cell tumour, Yolk sac tumour, Endodermal sinus tumour and dysgerminomas. ${ }^{10}$

The most important diagnostic tool for the diagnosis of ovarian tumours is USG with Doppler. Tumour markers also give additional information. CT is done in all cases of malignant ovarian tumours.

Surgery plays an important role in the treatment of tumours. Adequate surgery will confirm the diagnosis, exact histopathologic type and staging that help in the treatment

Fertility preservation is the important aspect of Surgery.

Normal appearing contralateral ovary along with tubes and uterus should be retained. If the contralateral ovary appears to be involved in the disease and fertility preservation is needed, it has to be removed as it may be a nidus for persistence of tumour. Unnecessary biopsy may result in adhesion which will interfere with fertility. Frozen section facility if not available, there is unnecessary loss of reproductive potential in young patients. $^{11}$

We had one case of immature teratoma grade 2 . It is graded according to the quantity of immature neural elements. The histological grading which is determined by the degree of immature neural elements is the most important factor in the survival. Grade 1 having less than one low power microscopic field and Grade 3 having more than three low power microscopic fields of these elements. Conservative staging and adjuvant BEP for all but stage IA grade 1 immature teratomas. ${ }^{11}$

In 2001, Pick et al reported close examination of tubal segments removed from women undergoing risk reducing bilateral salphingo oophorectomy in patients with strong history of ovarian cancer or had BRCA mutation. Of the 12 pathologic specimens, 6 had areas of cellular dysplasia noted in tubal epithelium and 5 additional specimens had hyperplastic lesions. These changes histologically resemble high grade serous ovarian cancer. ${ }^{12}$ In present study, patients with yolk sac tumour, Mucinous cystadeno carcinoma and immature teratoma fallopian tube did not show any significant pathology. 
There is increased rate of survival for the patients when malignant tumours are tackled by gynaec-oncologist. We had one case of TAH with BSO with infra colic omentectomy at 19 years of age. In these patients if facilities are available, they can opt for assisted reproductive techniques

Due to advances in assisted reproductive techniques, mature oocyte cryopreservation can be tried. But, it requires ovarian stimulation that may delay the cancer treatment. Ovulation induction for oocyte cryopreservation requires 2-3 weeks of exogenous hormone stimulation.

Ovarian tissue cryopreservation is the other method used. ${ }^{13}$ For optimal results, ovarian tissue is obtained prior to the initiation of cancer treatment laparoscopically and cryopreserved using slow freeze technique as follicular death and depletion of ovarian reserve can occur even after single dose of chemotherapy. The ovarian tissue is transplanted orthotopically into pelvis (grafting of ovarian cortex near the primary blood supply of the ovary) or heterotopic ovarian transplantation. Common sites of heterotopic transplantation are forearm and abdomen. Once the patient has survived her cancer frozen ovarian tissue is transplanted back to the patient. Live birth from orthotopically grafted ovarian tissue is reported. IVF is required for pregnancy to be achieved using oocyte from transplanted tissue.

\section{CONCLUSION}

Ovarian tumors even though rare in this age group, proper analysis should be done to rule out malignancy. Conservative surgery along with preservation of reproductive potential is an essential aspect of therapy.

Funding: No funding sources

Conflict of interest: None declared

Ethical approval: The study was approved by the Institutional Ethics Committee

\section{REFERENCES}

1. Consolidated report of hospital based cancer registries: 2012-2014. Avialable from: http:// ncrpindia.org/ALL_NCRP_REPORTS/HBCR_REP ORT_2012_2014/index.htm.

2. Devi KU. Current status of gynecological cancer care in India. J Gynecol Oncol. 2009;20(2):77-80.
3. Grovas A, Fremgen A, Rauck A, Ruyman D, Hutchinson C, Winchester D, et al. The national cancer data base report on patterns of childhood cancers in the United States. Cancer. 1997;80:2321.

4. Deligeoroglou E, Eleftheriades M, Shiadoes V. Ovarian masses during adolescence: clinical, ultrasonographic and pathological findings, serum tumour markers, and endocrinological profile. Gynaecol Endocrinol. 2004;19:1-8.

5. Rathore R, Sharma S, Arora D. Spectrum of childhood and adolescent ovarian tumours in India: 25 Years' experience at a single institution. Maced J Med Sci. 2016;4(4):551-5.

6. Chellamma VK. Adolescent Ovarian 2011 KFOG Vol.5 No:3 P 3-5 5,7

7. Bhattacharya NK, De A. Ovarian tumours in pediatric age group- A clinic-patho study of 10 years cases in West Bengal India. Indian J Med Paediatr Oncol. 2010;31(2):54-57.

8. Amatya A, Rana A, Gurung G. Ovarian tumours in childhood and adolescents- our eight years experiences. NJOG. 2008;3(1):39-42.

9. Choudry A, Bangash N, Malik A, Choudry H. A clinico pathological review of 15 cases 2008. J Ayub Med coll Abbottabad. 2008;20(4):18-21.

10. Hernon M, McKenna J, Busby G, Sanders C, Garden A. The histology management of ovarian cysts found in children and adolescent presenting to a children hospital from 1991 to 2007. BJOG. 2010;117(2):181-4.

11. Chitrathara K, Rajaram S, Maheswari A. Ovarian Cancer: comprehensive and contemporary management. $1^{\text {st }}$ ed. Jaypee Brothers Medical Publishers (P) Ltd.; 2009:121-122.

12. Piek JM, Verheijen RH, Kenemans P, Massuger LF, Bulten $\mathrm{H}$, van Diest PJ. BRCA 1/2-related ovarian cancers are of tubal origin A hypothesis. Gynecol Oncol. 2003;90;491

13. Gracia CR, Chang J, Kondapalli L, Prewitt M, Carlson CA, Mattei $P$, et al. Ovarian tissue cryopreservation for fertility preservation in cancer patients: Successful establishment and feasibility of a multidisciplinary collaboration. J Assist Reprod Genetics. 2012;29(6):495-502.

Cite this article as: Priya V. Clinicopathological profile of ovarian tumors in the age group 10-20 years. Int J Reprod Contracept Obstet Gynecol 2017;6:877-80. 